\title{
Self-Care Management Practices of Diabetic Patients Type 2 in Saudi Arabia
}

\author{
Budour Bandar ALotaibi \\ Prince Sultan Military Medical City, Riyadh, KSA \\ Email: Botaibi@psmmc.med.sa
}

How to cite this paper: ALotaibi, B.B. (2020) Self-Care Management Practices of Diabetic Patients Type 2 in Saudi Arabia. Open Journal of Nursing, 10, 1013-1025. https://doi.org/10.4236/ojn.2020.1011071

Received: September 13, 2020

Accepted: October 28, 2020

Published: October 31, 2020

Copyright $\odot 2020$ by author(s) and Scientific Research Publishing Inc. This work is licensed under the Creative Commons Attribution International License (CC BY 4.0).

http://creativecommons.org/licenses/by/4.0/

\begin{abstract}
Background \& Aim: Patients' self-care management practice in diabetes is considered a critical element to keep diabetes under control and the disease management is usually conducted by the patients themselves and/or their families. The main aim of this study is to assess the level of self-care management practices of diabetic patients type 2 in Saudi Arabia. Materials \& Methods: A cross-sectional study design was utilized in the Prince Sultan Military Medical City in Riyadh on a convenience sampling method consisted of 377 diabetic patients type 2 . The researcher used a self-administered questionnaire (Summery of Diabetes Self-care Activities) which was adopted from Toobert et al. (2000). Results: The study results revealed that the level of self-care management practices among patients with type $2 \mathrm{DM}$ is not satisfactory, except in the medication domain. Conclusion \& Recommendations: Policymakers should revise the education strategy for diabetic patients and develop health education campaigns that focus on the patient's family as well as the individual with type-2 DM.
\end{abstract}

\section{Keywords}

Diabetes, Self-Care, Management, Practices

\section{Introduction}

Diabetes is a leading cause of morbidity and mortality in the world, if undiagnosed or inadequately treated, multiple chronic complications leading to irreversible disability and death will be developed. The Kingdom of Saudi Arabia is not excluded from the global epidemic of diabetes mellitus, since it is the most challenging health problem facing this country [1]. According to the Saudi Arabian Ministry of Health, cases with diabetes mellitus are increasing from 1992 to 2015; in which 2.7 times increase in the incidence rates in less than two decades. 
In 2018 , there were 730,775 patients with diabetes attended the family and medical clinics across Saudi Arabia [2].

The increasing burden of diabetes in the Kingdom of Saudi Arabia is due to various factors, including a rising obesity rate and an aging population [2]. Management and treatment of diabetes is considered a lifelong issue and tends to be multi-dimensional and focuses on achieving better control of blood sugar levels [3]. More importantly, patients' self-care management practice in diabetes is considered a critical element to keep diabetes under control and about $95 \%$ of the disease management is usually conducted by the patients themselves and/or their families [4].

Self-care practices include regular physical activity, appropriate dietary practices, foot care practice, self-monitoring of blood glucose, and compliance with the treatment regimen [5]. Self-care activities, such as increasing physical activity and maintaining healthy nutrition, can slow disease progression [6]. In addition, compliance to treatment guidelines helps the patient achieve target glucose level and reduces the risk of diabetic complications and mortality; however, diabetes self-care measures demand a high level of motivation and consistent efforts from the patients [7].

Diabetes mellitus is a group of metabolic disorders which involve hyperglycemia due to decreased secretion of insulin, or decrease in the action of it or both [8]. Type-2 diabetes mellitus is known as adult-onset diabetes, which involves increase in the level of blood glucose, resistance in the action of insulin, as well as decrease in the level of insulin [9].

In this study, diabetic patients' self-care management practices have been defined as the total score of diabetic patients' answers on the questionnaire, which reflect self-care activities. These activities include: performing activities such as eating a healthy diet, physical activity in a regular pattern, conducting foot care, adherence to medication, and self-monitoring of blood glucose, the higher score indicates better self-care management practices and vice versa for the lower scores. Although the importance of diabetes self-care management practices is still effective and efficient in producing significant prevention and control of diabetes, findings of previous studies in Saudi Arabia were confirmed that the aspects of self-care management practices were more problematic, in which the study of Al Johani revealed that only $15.0 \%$ of diabetic patients had a control in their blood glucose level, and the compliance to self-management practices is low [10]. Therefore, the main aim of this study is to assess the level of self-care management practices of diabetic patients type- 2 in Saudi Arabia.

\section{Materials and Methods}

\subsection{Study Design, Setting, Sample, and Sampling}

The design of this study was quantitative descriptive cross-sectional. Cross-sectional designs are especially appropriate for describing the status of phenomena or relationships among phenomena at a fixed point. This type of design is useful for 
describing the study construct because it's suitable in terms of people, resources and it is relatively practical and manageable. The current study was carried out in the Prince Sultan Military Medical City (PSMMC) in Riyadh, Saudi Arabia in the period between March and July, 2019. The study population consisted of diabetic patients type- 2 who are attending diabetic treatment center and family and community medicine department in the PSMMC.

A convenience sampling method was applied to recruit the patients from diabetic patients type 2 in the PSMMC. Sample calculation was done by Stephen Thompson formula and with consultation of statistician [ $\mathrm{N}=38282, \mathrm{CI}=95.0 \%$, confidence limit $=0.05$, power $=0.080$ ]. Sample after calculation is 381 diabetic patients, in which 377 out of (98.9\%) have responded in the participation in the current study.

Male and female diabetic patients with type 2 who have uncontrolled diabetes mellitus, those with age 30 - 65 and older and who are attending PSMMC and those who have interested to participate in the current study; have been included. Patients with type 1 diabetes, and who are under 30 years and have mental health problems as well as the patient with physical disability have been excluded from the participation in the current study.

\subsection{Study Instrument, Scoring, and Statistical Analysis}

Summary of Diabetes Self-Care Activities (SDSCA) instrument which was adopted from Toobert et al. [11] was used in the present study. This instrument was translated into the Arabic language by AlJohani et al. [12], and it is valid for the use among Saudi population. The questionnaire consists of 4 parts: diet (2 items), exercise ( 2 items), blood glucose testing ( 2 items) and foot care ( 2 items) and 2 questions each about medications and smoking. This instrument is valid and reliable for the application, in which, the Cronbach's alpha was 0.76, split-half was 0.9, and the Cronbach's alpha scores for the instrument parts were ranging from good to excellent as: diet 0.89 , blood glucose testing 0.92 , exercise 0.83 , and foot care 0.77 [12].

The questionnaire also has an extension, and the items included in the extension to the SDSCA aim to identify the amount of self-management diabetes education the participants have received. The extension consists of 4 sections: diet (8 items), exercise (6 items), blood glucose testing (5 items) and medications (5 items). This part has some questions related to the advice given by the health care providers regarding self-care practices. The scale of the questionnaire ranges from 0 to 7 which reflect the extent to perform the specified self-care practices in days, in which the number of "0" reflects that the patient did not follow self-care practices in the last seven days, and the number 1 to 7 reflects the number of days in the last week that the patient conducted self-care practices.

General diet was measured as the mean number of days for items included in the diet, Specific diet was measured as the mean number of days for items included in the specific diet. Exercise was measured as the mean number of days 
for items included in the exercise. Blood-glucose testing was measured as the mean number of days for items included in the blood-glucose testing. Foot-care was measured as the mean number of days for items included in the foot care. Smoking status was measured as the number of cigarettes smoked per day. Additional items such as diet, medication, and foot care were measured as the total number of days. Statistical Package for Social Sciences (SPSS) version 22 was used to analyze data. Ethical approval was gathered from Institutional Review Board (IRB). Eligible subject who agrees to take apart from the study has been asked to provide consent. Participants have been informed that their participation is voluntary and they have the right to withdraw from the study at any time without any interference with their treatment and care plan. They have been also notified that there is no risk of harm to participate in the study as well as no benefits.

\section{Results}

In the current study, 377 respondents have participated. Demographic characteristics of the study participants showed that more than half (59.4\%) of the participants in the current study are females, while $40.6 \%$ of them are males. Also, the mean of patients; age is 58.19 years old; after classification of their ages, the table shows that $38.7 \%$ of the study participants are more than 60 years old, $36.1 \%$ are between 51 - 60 years old, and $21.0 \%$ are between $40-50$ years old. Regarding the educational characteristics of the patients, $42.2 \%$ of the study participants are illiterate, and $31.3 \%$ have below secondary, while $13.8 \%$ of them have a university degree. In addition, $81.7 \%$ of the study participants are not working, while $18.3 \%$ of them are working. Moreover, $51.2 \%$ of the study participants have an income of between 5000 - 10,000 Saudi Arabia Riyal (SAR), $27.1 \%$ of them have income below 5000 SAR, and less, while $21.0 \%$ of them have income more than 10,000 SAR. Moreover, the mean of duration of disease among patients is 14.50 years; after classification into groups, the table shows that $42.2 \%$ of the study participants have the diabetes for 15 years and more, $28.4 \%$ have diabetes for $5-10$ years, and $15.6 \%$ have diabetes for $11-15$ years (Table 1).

Regarding the mean and mean percentage of self-care management practices, the total mean of following a good diet for diabetic patients is 3.53 days (50.42\%), the total mean of days of following good exercise among diabetic patients is 3.15 days $(45.0 \%)$, the total mean of days of conducting foot care practices is 2.87 days ( $41.0 \%)$. In addition, the total mean of days of following medication practices is 5.54 days $(79.14 \%)$, while he total mean of days of testing blood glucose is 2.87 days [41.0\%] (Table 2).

Regarding the mean percentage of smoking practices of diabetic patients, more than half $(52.5 \%)$ of diabetic patients in the current study have not engaged in smoking since more than two years ago, $6.4 \%$ of them have engaged in smoking within the last month, and $1.6 \%$ have engaged in smoking one to two years ago (Table 3 ). Regarding the frequency and percentages of advice received 
Table 1. Demographic characteristics of the study participants $(n=377)$.

\begin{tabular}{|c|c|c|}
\hline Variables & Number & Percentage (\%) \\
\hline \multicolumn{3}{|l|}{ Gender } \\
\hline Male & 153 & 40.6 \\
\hline Female & 224 & 59.4 \\
\hline \multicolumn{3}{|l|}{ Age groups } \\
\hline$<40$ years & 16 & 4.2 \\
\hline $40-50$ years & 79 & 21.0 \\
\hline $51-60$ & 136 & 36.1 \\
\hline$>60$ years & 146 & 38.7 \\
\hline Mean \pm SD & & \\
\hline \multicolumn{3}{|l|}{ Educational level } \\
\hline Illiterate & 159 & 42.2 \\
\hline Below secondary & 118 & 31.3 \\
\hline Secondary school & 48 & 12.7 \\
\hline University and higher & 52 & 13.8 \\
\hline \multicolumn{3}{|l|}{ Working status } \\
\hline Working & 69 & 18.3 \\
\hline Not & 308 & 81.7 \\
\hline \multicolumn{3}{|l|}{ Level of income } \\
\hline$<5000$ & 102 & 27.1 \\
\hline $5000-10,000$ & 193 & 51.2 \\
\hline$>10,000$ & 79 & 21.0 \\
\hline Missing & 3 & 0.8 \\
\hline \multicolumn{3}{|l|}{ Duration of disease } \\
\hline$<5$ years & 52 & 13.8 \\
\hline $5-10$ years & 107 & 28.4 \\
\hline $11-15$ years & 59 & 15.6 \\
\hline$>15$ years & 159 & 42.2 \\
\hline Mean \pm SD & \multicolumn{2}{|c|}{$14.50 \pm 8.45$} \\
\hline Total & 377 & 100.0 \\
\hline
\end{tabular}


Table 2. Self-care management practices among diabetic patients regarding nutrition, exercise.

\begin{tabular}{cccccc}
\hline Domain & Max. Mean (\%) & Min & Mean & SD & Mean \% \\
\hline Diet (days) & $7(100.0)$ & $0(0.0)$ & 3.53 & 2.31 & 50.42 \\
Exercise (days) & $7(100.0)$ & $0(0.0)$ & 3.15 & 2.30 & 45.0 \\
Foot care within the last 7 days (days) & $7(100.0)$ & $0(0.0)$ & 2.87 & 1.21 & 41.0 \\
Medication practices (days) & $7(100.0)$ & $0(0.0)$ & 5.54 & 1.37 & 79.14 \\
Testing blood glucose (days) & $7(100.0)$ & $0(0.0)$ & 2.87 & 2.66 & 41.0 \\
\hline
\end{tabular}

The maximum mean score is 7.0 (each advice has 1 score if given and zero if not).

Table 3. Smoking practices among diabetic patients.

\begin{tabular}{ccc}
\hline Smoking & Number & $\%$ \\
\hline Never smoked & 198 & 52.5 \\
One to two years ago & 6 & 1.6 \\
Four to twelve months ago & 3 & 0.8 \\
One to three months ago & 2 & 0.5 \\
Within the last month & 24 & 6.4 \\
Missing & 144 & 38.2 \\
Total & 377 & 100.0 \\
\hline
\end{tabular}

from healthcare providers related to diet, $91.0 \%$ of the patients did not receive advice related to eating very few sweets, $74.0 \%$ of them also did not receive advice related to eating lots of food high in dietary fibres, and $72.4 \%$ did not receive advice related to reducing the number of calories to lose weight. On the other hand, $44.3 \%$ of the patients in the current study stated that they have received advice related to following a complex carbohydrate diet, and $38.2 \%$ have received advice related to following a low-fat eating plan. The total mean percentage of received medical advice related to diet is $28.57 \%$ (Table 4).

Moreover, $94.2 \%$ of the patients did not receive advice related to fit exercise into your daily routine, $82.5 \%$ of them did not receive advice related to exercising continuously for at least 20 minutes at least 3 times a week, and $68.0 \%$ did not receive advice related to engaging in a specific amount, type, duration and level of exercise. On the other hand, $48.0 \%$ of the patients in the current study have received advice related to getting low level exercise on a daily basis, 32.0\% have received advice related to engaging in a specific amount, type, duration and level of exercise. The total mean percentage of received medical advice related to exercising is $17.0 \%$. Additionally, $78.0 \%$ of the patients have received advice related to testing their blood sugar using a drop of blood from their finger and a colour chart and $10.9 \%$ of them have received advice related to testing their blood sugar using a machine to read the results. The total mean percentage of received medical advice related to testing blood sugar is $25.0 \%$ (Table 5 \& Table 6). The mean percentage of self-care management practices for patients with type-2 DM in this study was $51.31 \%$, which is not satisfactory. 
Table 4. Frequency and percentages of advice related to diet.

\begin{tabular}{lccc}
\hline No & Advice related to Diet & No (\%) & Yes (\%) \\
\hline 1 & Follow a low-fat eating plan & $233(61.8)$ & $144(38.2)$ \\
2 & Follow a complex carbohydrate diet & $210(55.7)$ & $167(44.3)$ \\
3 & Reduce the number of calories you eat to lose weight & $273(72.4)$ & $104(27.6)$ \\
4 & Eat lots of food high in dietary fibres & $279(74.0)$ & $98(26.0)$ \\
5 & Eat lots of fruits and vegetables & $256(67.9)$ & $121(32.1)$ \\
6 & Eat very few sweets & $343(91.0)$ & $34(9.0)$ \\
7 & Others & $28(76.4)$ & $89(23.6)$ \\
& Mean \pm SD & $2.00 \pm 1.27$ \\
\end{tabular}

Table 5. Frequency and percentages of advice related to exercise.

\begin{tabular}{|c|c|c|c|}
\hline No & Advice related to Exercise & No (\%) & Yes (\%) \\
\hline 1 & Get low level exercise on a daily basis & $196(52.0)$ & $181(48.0)$ \\
\hline 2 & $\begin{array}{c}\text { Exercise continuously for at least } 20 \text { minutes } \\
\text { at least } 3 \text { times a week }\end{array}$ & $311(82.5)$ & $66(17.5)$ \\
\hline 3 & Fit exercise into daily routine & $355(94.2)$ & $22(5.8)$ \\
\hline 4 & $\begin{array}{l}\text { Engage in a specific amount, type, } \\
\text { duration and level of exercise }\end{array}$ & $204(68.0)$ & $96(32.0)$ \\
\hline 5 & Others & $255(94.2)$ & $22(5.8)$ \\
\hline & Mean \pm SD & \multicolumn{2}{|c|}{$0.85 \pm 0.77$} \\
\hline & Mean \% & \multicolumn{2}{|c|}{$17.0 \%$} \\
\hline
\end{tabular}

Table 6. Frequency and percentages of advice related testing sugar $(n=375)$.

\begin{tabular}{|c|c|c|c|}
\hline No & Advice related to Testing Sugar & No (\%) & Yes $(\%)$ \\
\hline 1 & $\begin{array}{c}\text { Test your blood sugar using a drop of blood from } \\
\text { your finger and a colour chart }\end{array}$ & $81(21.5)$ & $294(78.0)$ \\
\hline 2 & Test blood sugar using a machine to read the results & $334(88.6)$ & $41(10.9)$ \\
\hline 3 & Test urine for sugar & $364(96.6)$ & $11(2.9)$ \\
\hline 4 & Others & $344(91.2)$ & $31(8.2)$ \\
\hline & Mean \pm SD & \multicolumn{2}{|c|}{$1.00 \pm 0.28$} \\
\hline & Mean \% & \multicolumn{2}{|c|}{$25.0 \%$} \\
\hline
\end{tabular}




\section{Discussion}

The current study results revealed that the total mean of following a good diet for diabetic patients is 3.53 days out of 7 days $(50.42 \%)$, this score is at margin level, these results are not similar to the results of previous studies [13] [14] [15] which reported that low mean score of diet, and not similar to the results of the studies conducted by the [16] [17] showed that high mean score of diet. Moreover, the results of the current study are consistent with the results of $\mathrm{Al}$ Johani et al. [10] which revealed that total mean of following a healthy diet for diabetic patients is 3.60 days in Saudi Arabia. The differences in the mean level of following a healthy diet between the current study results and previous study results could be attributed to the differences in the culture of patients included. In addition, the differences could be attributed to the differences in the sample and sampling method.

On the other hand, similarity with the study of $\mathrm{Al}$ Johani et al. [10] could be attributed to the fact that both participants for both studies are from the same culture (Saudi Arabia). In the Arab context, there is a high probability that traditional social and cultural practices work against the efforts made by people with type-2 diabetes mellitus to maintain an optimal diet [10]. Regarding following exercise, the total mean of days of following good exercise among diabetic patients is 3.15 days (45.0\%), which is considered low level. These results are not consistent with the results of Dedefo et al. [18], which revealed that $63.5 \%$ of the patients had adequate exercise management. In addition, these results are not consistent with the results of $\mathrm{Al}$ Johani et al. [10], which revealed that only $53 \%$ of the sample reported that they exercised three or more days per week as recommended.

Additionally, the percentage of the current study is higher than that reported in similar Asian studies. In a recent Chinese study, it was reported that $40 \%$ of participants who had type 2 diabetes mellitus maintained healthy exercise practices throughout the week [19], and it was lower than that reported in another Korean study, in which 54\% of Korean people with type 2 diabetes mellitus who visited a general practitioner maintained good exercise practices [20]. Moreover, the results of previous studies [13] [15] [16] [17] [21] showed that low mean score of exercise. Differences could be attributed to the type of sample and differences in the mean age of the study sample, in which the age of patients play an important role in the process of exercise.

A possible reason for the large proportion of participants in the current study not exercising is that $74.8 \%$ were over 50 years of age, and $38.7 \%$ were over 60 years old. They may not be able to perform regular exercise due to general physical decline or poor health. This may be due to a lack of places for exercise and a lack of suitable environments in Saudi Arabia for activities such as walking. The hot weather in Saudi Arabia is a further potential factor contributing to low physical exercise rates among the population in Saudi Arabia. In addition, Saudi Arabian culture is quite unlike Asian culture in that it does not encourage 
other types of healthy activities for older people such as yoga and tai chi [10].

The current study results revealed that the mean of days in which the patients wash feet is 3.07 days out of 7 (43.86\%), soak feet in at least 30 minutes of physical activity within the last 7 days is 0.85 days out of 7 days (12.14\%), dry between toes after washing 6.74 days $(96.29 \%)$, check feet 0.64 days $(9.14 \%)$, inspect the inside of shoes 3.08 days (44.0\%). The total mean of days of conducting foot care practices is 2.87 days (41.0\%), this level is considered low level. These results are similar to the previous study results of [10] [15] [17], which showed that low mean score of foot care. In contrast to the Bariyyah et al. [16] and Daoud et al. [21], which reported that the foot care, had high mean score. On the other hand, the results of the current study are lower from what has been reported by Dedefo et al. [18] in Ethiopia, in which the majority of (82.9\%) of patients had adequate foot care.

Moreover, the results of the current study are lower from what has been revealed by $\mathrm{Al}$ Johani et al. [10] in which the mean of days of the patients' compliance to foot care is 3.49. Low level of foot care among patients in the current study could be attributed to the large proportion of patients who are illiterate (42.2\%), and $31.3 \%$ are below secondary, this issue can predict low self-care management because they simply do not know and understand the importance and the process how to take care of their foot, this could be attributed to the lack of health education and lack of care introduced from their care giver or families.

The current study results revealed that the mean of days in which the patients take recommended diabetes medication is 6.81 days out of 7 (97.29\%), take recommended insulin injections 4.48 days out of 7 days (64.0\%), and take recommended number of diabetes pills 5.32 days $(76.0 \%)$. The total mean of days of following medication practices is 5.54 days $(79.14 \%)$. The level of compliance of following medication practices by the patients is considered very well, this could be attributed to the fact that the patients are afraid of not taking their medication due to its fatal complication if not taken. These results are somewhat consistent with the results of Al Johani et al. [10] in Saudi Arabia, which revealed that the total mean of days of taking medication is 6.26. The patients in general (especially not educated) understand that the management of disease is only by taking medication. In the current study, taking medication was the most commonly practiced form of self-care reported among patients, which clearly reflects a high level of dependence on medication.

The current study results were consistent with the study of Alsomali [22] in Saudi Arabia, which showed that taking of medication among people with type 2 DM was prioritized over other diabetes self-care activities, as it is an easy task, and patients face fewer challenges taking medication. Moreover, this finding was consistent with the findings of other studies, which found high levels of adherence to medication such as Tewahido \& Berhane [23]. A similar study carried out by Al-Shehri [15] in Saudi Arabia also found that patients' commitment to adherence to medication was the highest among self-care activities. Regarding 
self-care management of testing blood glucose practices, the current study results revealed that the mean of days in which the patients test their blood glucose is 3.14 days out of 7 (44.85\%), and the average days of testing, blood sugar in terms of times recommended by health care provider is 2.58 days out of 7 days (36.85\%). The total mean of days of testing blood glucose is 2.87 days $(41.0 \%)$, this considered low level. These results are higher than what has been revealed by Dedfo et al. [18], in which $15.1 \%$ of diabetic patients had adequate blood glucose testing practices. The largest group of respondents was those who did not test blood glucose on any of the previous seven days (35.3\%). On the other hand, the current study results are lower from what has been revealed by Alsomali [22], which revealed that more than half of the patients tested their blood sugar on two days in the last week or less.

These low levels, which revealed in the current study, could be explained by the absence of recommendations from healthcare providers regarding testing blood sugar, or a recommendation on the frequency of testing their blood sugar. Similar studies suggest that blood sugar testing levels are generally low among diabetes patients, despite this being an essential part of diabetes control [15]. The patients in the current study are aware of the importance of testing their blood sugar level to manage their diabetes, and participants tested their blood sugar at times when they had eaten too much or were not sure if they had taken the correct medication dose. An explanation of this low adherence level regarding testing blood glucose could be the difficulties in using the glucometer at home; many patients have technical difficulties in testing their blood sugar levels, especially those aged 50 and above. Another explanation could be attributed to the expensive status of the glucometer especially its sticks which prevent the patients from buying these sticks.

The current study results revealed that more than half (52.5\%) of diabetic patients engaged in smoking since more than two years ago, or never smoked, 6.\% of them have engaged in smoking within the last month, and $1.6 \%$ have engaged in smoking one to two years ago. meaning that $8.5 \%$ of the patients are smoking. These results are lower from what has been by Noubiap et al. [24], which revealed that $12.9 \%$ of patients are smokers, and it was lower from what has been revealed by Reynolds et al. [25], which revealed that $40.3 \%$ of the patients are smokers. Low level of smoking among patients in the current study could be attributed to the Islamic approach within Saudi Arabia, in which smoking is forbidden in Islamic culture and religion. Moreover, low percentage of smoking among patients in the current study could be attributed to the health education received by the patients and their health literacy in recent years since smoking interferes with insulin resistance and aggravates the micro- and macro-vascular complications of diabetes mellitus [26].

\section{Conclusion}

The study results revealed that the level of self-care management practices among patients with type $2 \mathrm{DM}$ is not satisfactory, except in the medication do- 
main. Health care providers should begin by taking time to evaluate their patients' perceptions and make realistic and specific recommendations for self-care activities. In addition, healthcare providers need to provide guidance and a treatment plan uniquely suited to each individual patient. Policymakers should revise the education strategy for diabetic patients and develop health education campaigns that focus on the patient's family as well as the individual with type 2 DM. This can be achieved by health education presentations for the families of patients in order to improve their awareness about DM management. Future research needs to examine the relationship between other variables and diabetes self-care practices among Saudi adults with type 2 DM by using a mixed methods approach.

\section{Limitations of the Study}

The study focused only on quantitative approach for describing and analysing issues pertaining to self-care management practices, mixed design often have border view than single approach. In addition, the qualitative approach focused on the hidden issues related to self-care practices, which were not appeared in the study questionnaire.

\section{Conflicts of Interest}

The author declares no conflicts of interest regarding the publication of this paper.

\section{References}

[1] Alotaibi, A., Perry, L., Gholizadeh, L. and Al-Ganmi, A. (2017) Incidence and Prevalence Rates of Diabetes Mellitus in Saudi Arabia: An Overview. Journal of Epidemiology and Global Health, 7, 211-218.

https://doi.org/10.1016/j.jegh.2017.10.001

[2] Ministry of Health. Statistics Report 2015. http://www.moh.gov.sa/en/Ministry/Statistics/book/Pages/default.aspx

[3] Srinatha, K., Basavegowdab, M. and Tharunic, N. (2017) Diabetic Self-Care Practices in Rural Mysuru, Southern Karnataka, India-A Need for Diabetes Self Management Educational (DSME) Program. Diabetes \& Metabolic Syndrome: Clinical Research \& Reviews, 11, S181-S186. https://doi.org/10.1016/j.dsx.2016.12.029

[4] Mariye, T., Tasew, H., Teklay, G., Gerensea, H. and Daba, W. (2018) Magnitude of Diabetes Self Care Practice and Associated Factors among Type Two Adult Diabetic Patients Following at Public Hospitals in Central Zone, Tigray Region, Ethiopia, 2017. BMC Research Notes, 11, 380. https://doi.org/10.1186/s13104-018-3489-0

[5] Raithatha, S.J., Shankar, S.U. and Dinesh, K. (2014) Self-Care Practices among Diabetic Patients in Anand District of Gujarat. ISRN Family Medicine, 2014, Article ID: 743791. https://doi.org/10.1155/2014/743791

[6] Shrivastava, S.R., Shrivastava, P.S. and Ramasamy, J. (2013) Role of Self-Care in Management of Diabetes Mellitus. Journal of Diabetes \& Metabolic Disorders, 12, 14. https://doi.org/10.1186/2251-6581-12-14

[7] Polonsky, W.H. and Henry, R.R. (2016) Poor Medication Adherence in Type 2 
Diabetes: Recognizing the Scope of the Problem and Its Key Contributors. Patient Preference and Adherence, 10, 1299-1307. https://doi.org/10.2147/PPA.S106821

[8] American Diabetes Association (2012) Diagnosis and Classification of Diabetes Mellitus. Diabetes Care, 35, S64-S71. https://doi.org/10.2337/dc12-s064

[9] National Institute of Diabetes and Digestive and Kidney Diseases (2014) Diagnosis of Diabetes and Prediabetes.

[10] Al Johani, K., Kendall, G. and Snider, P. (2015) Self-Management Practices among Type 2 Diabetes Patients Attending Primary Health-Care Centres in Medina, Saudi Arabia. Eastern Mediterranean Health Journal, 21, 621-628. https://doi.org/10.26719/2015.21.9.621

[11] Toobert, D., Hampson, S. and Glasgow, R. (2000) The Summary of Diabetes Self-Care Activities Measure. Diabetes Care, 23, 943-950. https://doi.org/10.2337/diacare.23.7.943

[12] AlJohani, K.A., Kendall, G.E. and Snider, P.D. (2014) Psychometric Evaluation of the Summary of Diabetes Self-Care Activities-Arabic (SDSCA-Arabic): Translation and Analysis Process. Journal of Transcultural Nursing, 27, 65-72. https://doi.org/10.1177/1043659614526255

[13] Bonger, Z., Shiferaw, S. and Tariku, E. (2018) Adherence to Diabetic Self-Care Practices and Its Associated Factors among Patients with Type 2 Diabetes in Addis Ababa, Ethiopia. Patient Preference and Adherence, 12, 963-970.

https://doi.org/10.2147/PPA.S156043

[14] Kushwaha, A., Kumari, S. and Kushwaha, N. (2017) Self Care in Diabetes: A Study amongst Diabetics in an Urban Community. International Journal of Community Medicine and Public Health, 3, 293-298. https://doi.org/10.18203/2394-6040.ijcmph20151579

[15] Al-Shehri, F. (2014) Quality of Life among Saudi Diabetics. Journal of Diabetes Mellitus, 4, 225-231. https://doi.org/10.4236/jdm.2014.43032

[16] Bariyyah, N.K.M.H., Bujang, M.A., Baharum, N., Mastura, I. and Shah, S.A. (2018) Self-Care Activities among Diabetic Patients and Factors Affecting Glycaemic Control in Primary Health Care, Malaysia. Journal of Diabetes and Clinical Practice, 1, 103.

[17] Solhi, M., Nejaddadgar, N., Jegarghosheh, S., Abolfathi, M. and Ashtarian, H. (2017) Self-Care and Related Factors in Patients with Type 2 Diabetes. Asian Journal of Biomedical and Pharmaceutical Sciences, 7, 6-10.

[18] Dedefo, M.G., Ejeta, B.M., Wakjira, G.B., et al. (2019) Self-Care Practices Regarding Diabetes among Diabetic Patients in West Ethiopia. BMC Research Notes, 12, 212. https://doi.org/10.1186/s13104-019-4258-4

[19] Xu, Y., Pan, W. and Liu, H. (2010) Self-Management Practices of Chinese Americans with Type 2 Diabetes. Nursing \& Health Sciences, 12, 228-234. https://doi.org/10.1111/j.1442-2018.2010.00524.x

[20] Lee, H., Ahn, S. and Kim, Y. (2009) Self-Care, Self-Efficacy, and Glycemic Control of Koreans with Diabetes Mellitus. Asian Nursing Research, 3, 139-146. https://doi.org/10.1016/S1976-1317(09)60025-6

[21] Daoud, N., Osma, A., Hart, T., Barry, E. and Adler, B. (2014) Self-Care Management among Patients with Type 2 Diabetes in East Jerusalem. Health Education Journal, 74, 603-615. https://doi.org/10.1177/0017896914555038

[22] Alsomali, S. (2018) An Investigation of Self-Care Practice and Social Support of Patients with Type 2 Diabetes in Saudi Arabia. Published Thesis, University of Salford, 
School of Health and Society, Salford.

[23] Tewahido, D. and Berhane, Y. (2017) Self-Care Practices among Diabetes Patients in Addis Ababa: A Qualitative Study. PLoS ONE, 12, e0169062.

https://doi.org/10.1371/journal.pone.0169062

[24] Noubiap, J.J., Nansseu, J.R., Endomba, F.T., Ngouo, A., Nkeck, J.R., Nyaga, U.F., Bigna, J.J., et al. (2019) Active Smoking among People with Diabetes Mellitus or Hypertension in Africa: A Systematic Review and Meta-Analysis. Scientific Reports, 9, Article No. 588. https://doi.org/10.1038/s41598-018-37858-Z

[25] Reynolds, K., Liese, A.D., Anderson, A.M., Dabelea, D., Standiford, D., Daniels, S.R., Lawrence, J.M., et al. (2011) Prevalence of Tobacco Use and Association between Cardiometabolic Risk Factors and Cigarette Smoking in Youth with Type 1 or Type 2 Diabetes Mellitus. The Journal of Pediatrics, 158, 594-601.e1. https://doi.org/10.1016/j.jpeds.2010.10.011

[26] Chang, S.A. (2012) Smoking and Type 2 Diabetes Mellitus. Diabetes \& Metabolism Journal, 36, 399-403. https://doi.org/10.4093/dmj.2012.36.6.399 